\title{
Tailward propagation of Pi2 waves in the Earth's magnetotail lobe
}

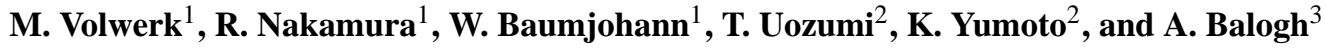 \\ ${ }^{1}$ Space Research Institute, Austrian Academy of Sciences, 8042 Graz, Austria \\ ${ }^{2}$ Space Environment Research Center, Kyushu University, Fukuoka 812-8581, Japan \\ ${ }^{3}$ Imperial College, London, SW7 2AZ, UK
}

Received: 14 July 2008 - Revised: 17 November 2008 - Accepted: 17 November 2008 - Published: 9 December 2008

\begin{abstract}
Pi2 waves are an intergral part of the substorm process and have been observed on the ground and in space. Using the special ability of Cluster to determine the propagation direction of signals measured in the magnetometer data, it is found that in the lobes of the Earth's magnetotail, for the cases in this study, the Pi2 waves are travelling tailward. The polarization of the waves in the lobes corresponds well with the polarization observed in the highest latitude ground station. The propagation velocity of the Pi2 waves in the lobes is basically Alfvénic.
\end{abstract}

Keywords. Magnetospheric physics (Magnetotail; MHD waves and instabilities)

\section{Introduction}

Pi2 waves have been studied for a long time, and are found to be an integral part of magnetic substorms, related to onset (see e.g. the review by Olson, 1999). They are described by periods between 40 and $150 \mathrm{~s}$, and mainly measured by ground magnetometer stations. The origin of these waves is still under scrutiny, however Shiokawa et al. (1998), Kepko and Kivelson (1999), and Kepko et al. (2001) have shown the relation between bursty bulk flows (BBFs), flow braking and the generation of Pi2 waves. Pashin et al. (1982), Lester et al. (1983), and Glaßmeier et al. (1988) discussed the origin of the Pi2 waves in relation to the substorm current wedge.

Lately, various papers have been published that combine wave measurements by spacecraft with Pi2 observations from ground stations. Keiling et al. (2001) and Kim et al. (2005) have used Polar data in the regions between $3.7 \leq L \leq 4.1 R_{E}$ and $4.2 \leq L \leq 8.5 R_{E}$, respectively. Sigsbee et al. (2002) have used Geotail data when the spacecraft was betwee $10 \leq L \leq 13 R_{E}$. One of their main findings was that

Correspondence to: $\mathrm{M}$. Volwerk

(martin.volwerk@oeaw.ac.at)

the Pi2 waves measured on the ground in the $B_{\mathrm{H}}$ component correlated rather well with the waves in the compressional component measured by the spacecraft. Collier et al. (2006) presented the first Pi2 observations by Cluster, where the spacecraft were in the plasmasphere or on the plasmapause, all at low $(\leq 6.6) \mathrm{L}$-shells. The middle to low latitude Pi2s were interpreted at cavity modes driven by a substorm. The higher latitude Pi2 waves were interpreted as transients associated with field-aligned currents.

Keiling et al. (2006) used Cluster observations in the lobe of the magnetotail, at large distance from Earth, to relate Pi2 pulsations and pulsed reconnection. Recently, Keiling et al. (2008) also used THEMIS data to look at Pi2 waves in the magnetotail at a distance of $\sim 8.3 R_{E}$, with the spacecraft again near the plasma sheet. In this paper it is shown that there is a correlation between substorm particle injection periodicity and the occurrence of Pi2 and modulation of the aurora with the same periodicity.

It is clear that similar wave forms in the Pi2 frequency interval can be seen in the tail and on the ground. In this paper we use the special ability of Cluster to determine the propagation direction of the waves in the tail. We will show three case studies in which the Pi2 waves are observed in the Earth's magnetotail lobe.

\section{The events}

We have searched for conjuctions of Cluster data obtained in 2001 with the 210 meridian chain, when Pi2 activity was measured on the ground. Out of the small set of conjunctions (only 5 events), three were chosen for this case study as all three of them had the Cluster spacecraft in the lobe of the magnetotail.

Published by Copernicus Publications on behalf of the European Geosciences Union. 


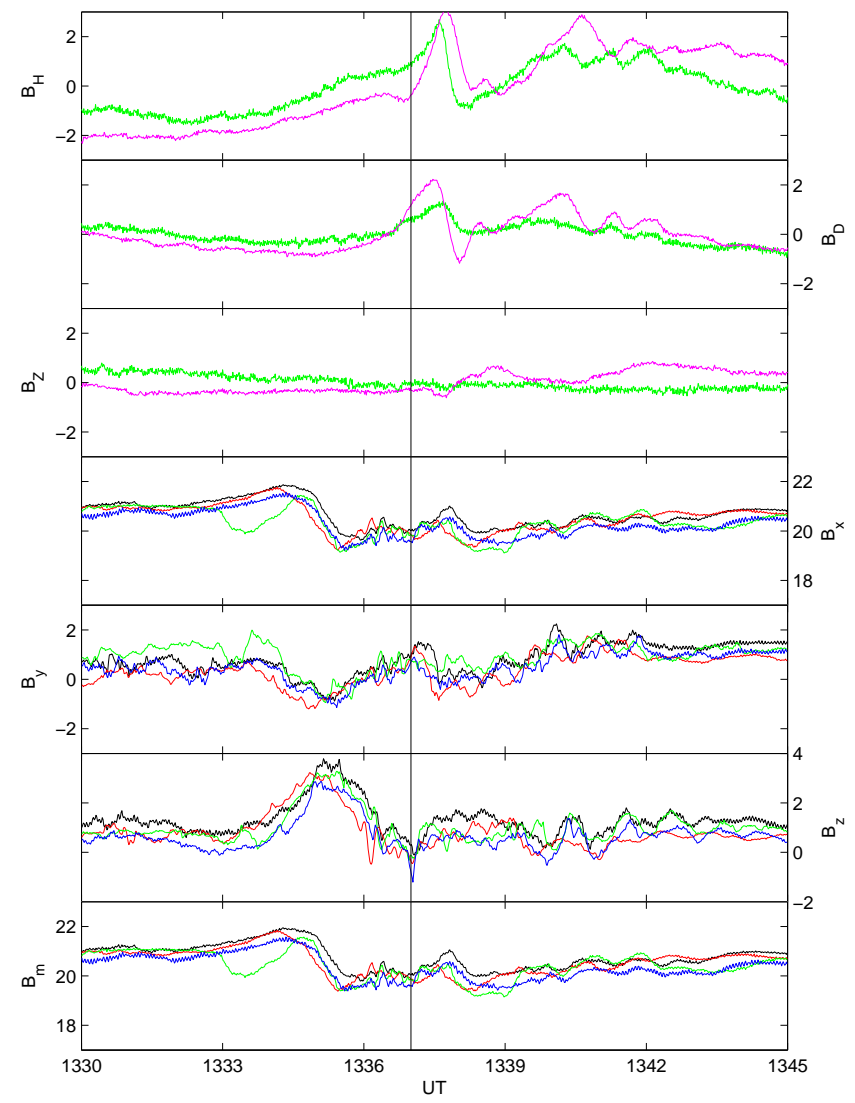

Fig. 1. Magnetic data for 24 August 2001. The top three panels show the H-, D- and Z-component of the magnetic field from the 210 meridian stations MSR (green) and CHD (magenta, divided by 2). The bottom three panels show the magnetic field measure by the Cluster spacecraft (in usual colours) in GSM coordinates. The dashed vertical line shows the time of Pi2 onset.

\subsection{Event 1: 24 August 2001}

The first event takes place on 24 August 2001. The Cluster spacecraft are located near $(-18.5,-2.3,4.3) R_{E}$. The automated procedure from the 210 meridian magnetometer chain (see e.g. Uozumi et al., 2000) found Pi2 onset at $\sim 13: 37$ UT. In Fig. 1 the magnetic field data of two 210 stations (midlatitude Moshiri, MSR, at GMlat $44^{\circ}$, GMlon $142^{\circ}$ and highlatitude Chokurdakh, $\mathrm{CHD}$, at GMlat $71^{\circ}$, GMlon $148^{\circ}$ ) are shown, as well as the magnetometer data from Cluster.

In order to investigate the possible $\mathrm{Pi} 2$ wave power measured by Cluster, the magnetic field data are transformed to a Mean Field Aligned (MFA) coordinate system, in which the two transverse components are combined into left- and right-hand polarized components $\left(B_{\mathrm{r}}=B_{\mathrm{t} 1}+i B_{\mathrm{t} 2}\right.$ and $\left.B_{1}=B_{\mathrm{t} 1}-i B_{\mathrm{t} 2}\right)$. A wavelet transform with Morlet mother wavelet (see e.g. Mallat, 1998) is used to study the wave power for $\mathrm{C} 1$ and the 210 stations. The Morlet wavelet is used as this wavelet can process complex data (Baumjohann et al., 1999). The wavelet spectrograms for $\mathrm{C} 1$ and the 210 stations are shown in Fig. 2.
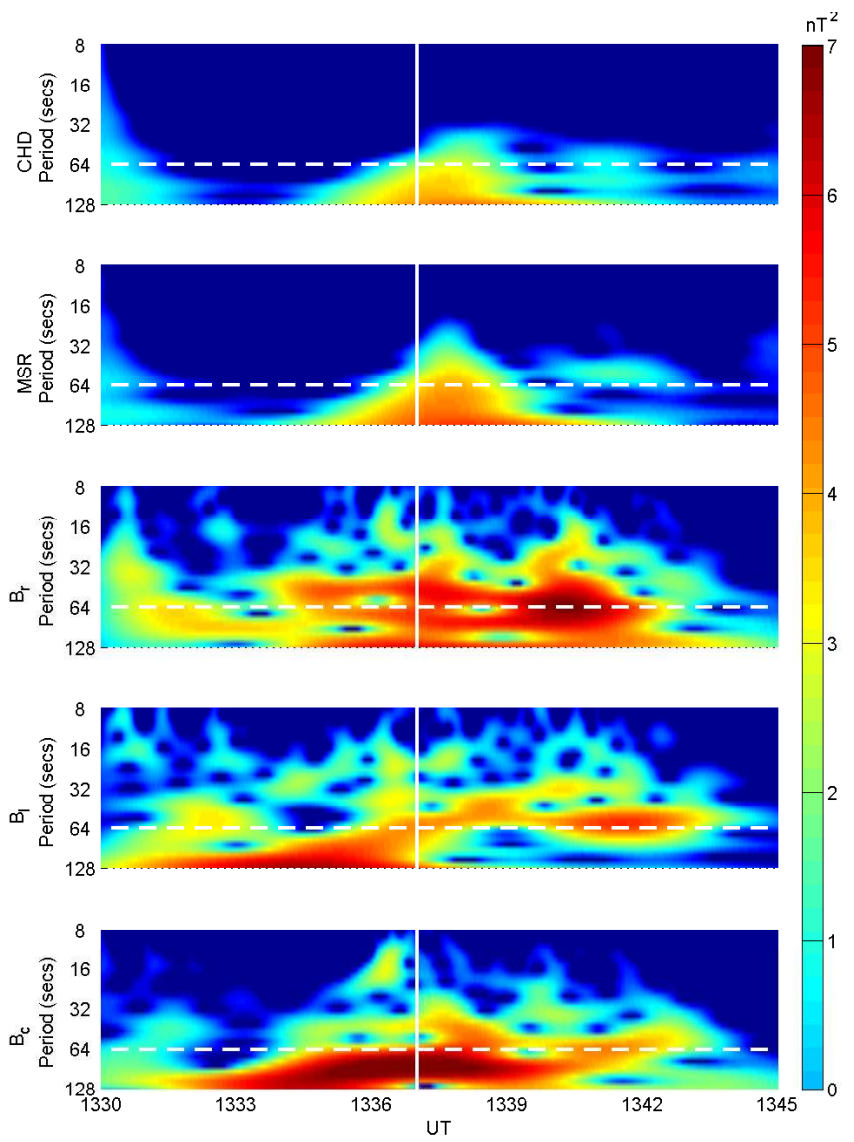

Fig. 2. Wavelet analysis for 24 August 2001 of the H-component from CHD and MSR and for the right- and left-hand polarized and compressional components of $\mathrm{C} 1$.

The vertical dashed line in Fig. 1 shows the time of Pi2 onset, and also devides the magnetic field signature measured by Cluster in two different parts. Before onset $(\sim 01: 33-$ $01: 37 \mathrm{UT})$, there is a strong signature in $B_{\mathrm{z}}$, wherease after onset ( 01:38-01:42 UT) there is a more oscillatory activity in the magnetic field. We will look separately at these two intervals. The first interval shows basically the same structure for all four Cluster spacecraft, except for a slight variation in $B_{\mathrm{x}}$ for $\mathrm{C} 3$ just after 01:33 UT. Timing analysis of the data (Harvey, 1998) with cross-correlating the data of all four spacecraft shows that this structure is moving with a velocity $\mathbf{v}_{\text {pulse }} \approx(590,-190,220) \mathrm{km} / \mathrm{s}$, which means that the structure is moving mainly Earthward.

Taking the location of the Cluster spacecraft at $X \approx-18.5 R_{E}$ and the velocity of the pulse, we find that it will reach the Earth in $\sim 200 \mathrm{~s}$, assuming constant velocity. The front of the pulse passes by the spacecraft at $\sim 13: 34$ UT, which means that it reaches Earth at $\sim 13: 37$ UT, which means at $\mathrm{Pi} 2$ onset as measured by the 210 meridian.

The wavelet analysis shows that the $\mathrm{Pi} 2$ waves appear in the MSR data just after $\sim 13: 40 \mathrm{UT}$ and that there is good 
correspondence with the Pi2 waves observed in the left-hand polarized component of $\mathrm{C} 1$ at the same time, albeit that the power in the waves observed on the ground is smaller than measured in space.

This second interval of the event, with the oscillating magnetic field (mainly visible in $B_{\mathrm{Z}}$ ) shows, after timing analysis, that at Cluster the Pi2 waves are travelling with a velocity $\mathbf{v}_{\mathrm{Pi} 2} \approx(-170,-17,-316) \mathrm{km} / \mathrm{s}$, which means they are travelling tailward and southward. Cross-correlation of the waves observed by Cluster and by the 210 meridian stations indicates a lag time between Cluster and 210 of $\Delta T \approx 25 \mathrm{~s}$ with 210 leading. With the observed velocity of the waves at Cluster, we find that the waves could have traveled up to $1.5 R_{E}$, thus they cannot have originated from the field lines connected to the 210 station MSR, assuming constant propagation velocity.

Note that the Pi2 waves on the ground are mainly in the $\mathrm{H}$ and D-component of the magnetic field. Polarization analysis for the interval 13:39-13:45 UT shows that on the ground the waves are almost linearly polarized at MSR and left-hand polarized at CHD. The latter fits well with the observed polarization of the Pi2 waves at Cluster. The foot points of Cluster map to latitude $\sim 73^{\circ}$ and longitude $\sim 196^{\circ}$, which means at approximately the same latitude as $\mathrm{CHD}$.

\subsection{Event 2: 6 October 2001}

The second event takes place on 6 October 2001. The Cluster spacecraft are located near $(-16.3,8.8,-5.1) R_{E}$. The automated procedure from the 210 meridian magnetometer chain found Pi2 onset at $\sim 13: 00$ UT. In Fig. 3 the magnetic field data of two 210 stations (MSR and CHD) are shown, as well as the magnetometer data from Cluster.

The vertical dotted line in Fig. 3 shows Pi2 onset on the ground. It is clear from the Cluster data that strong wave power in the Pi2 period band already occurs before ground onset. Wavelet analysis of the Cluster and 210 data shows some interesting features, see Fig. 4. First of all, at the ground stations the period of the main power at the highlatitude station CHD is longer than at the mid-latitude station MSR. Comparing the Cluster data with the 210 data around onset time, there is a correspondence in power between $B_{\mathrm{r}}$ and MSR at $\sim 66 \mathrm{~s}$ and between $B_{1}$ and CHD at $\sim 84 \mathrm{~s}$.

Timing analysis of the Cluster data shows that the waves are travelling at high velocity, $v_{\mathrm{Pi} 2} \approx(-224,670,-1737) \mathrm{km} / \mathrm{s}$, which means that they are travelling at the Alfvén speed, tailward and southward. Cross-correlation between the ground and space data shows that Cluster is leading by $\sim 86 \mathrm{~s}$.

Approximately 5 min later, at $\sim 13: 09 \mathrm{UT}$, there is another activation of wave power in the MSR data, at slightly longer wave period. This same power shows up as compressional power in the Cluster wavelet spectra. Timing analysis on the Cluster data shows that the waves are travelling at a velocity $v_{\mathrm{Pi} 2} \approx(-240,688,-1727) \mathrm{km} / \mathrm{s}$, only slightly different from

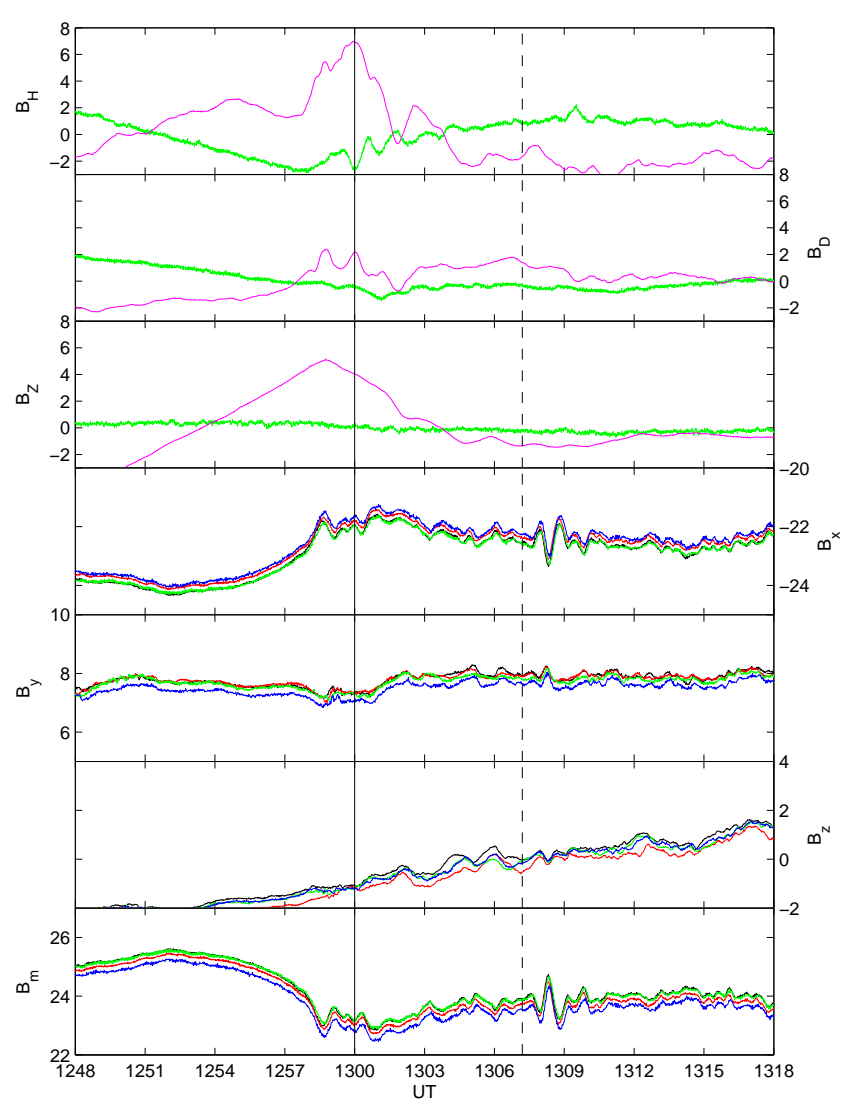

Fig. 3. Magnetic data for 6 October 2001. The top three panels show the H-, D- and Z-component of the magnetic field from the 210 meridian stations MSR (green) and CHD (magenta, divided by 10)). The bottom three panels show the magnetic field measure by the Cluster spacecraft (in usual colours) in GSM coordinates. The dashed vertical line shows the time of Pi2 onset.

the previous waves and again Alfvénic. Cross-correlation between the ground and space data shows that Cluster is leading by $71 \mathrm{~s}$.

The magnetometers on the ground show that the Pi2 waves at MRS are very elliptically left-hand polarized, and CHD also shows very elliptical left-hand polarized waves. In this event, the corresponding waves in the Cluster data was mainly in the compressional component.

\subsection{Event 3: 26 July 2001}

The third event takes place on 26 July 2001 . The Cluster spacecraft are located near $(-12.5,11.2,7.2) R_{E}$. The automated procedure from the 210 meridian magnetometer chain found Pi2 onset at $\sim 19: 13$ UT. In Fig. 5 the magnetic field data of two 210 stations (MSR and CHD) are shown, as well as the magnetometer data from Cluster. This is the most complicated of the three events.

Transforming the Cluster data to MFA coordinates again, we perform a wavelet transform to study the wave power for $\mathrm{C} 1$ and some 210 stations, which is shown in Fig. 6. 

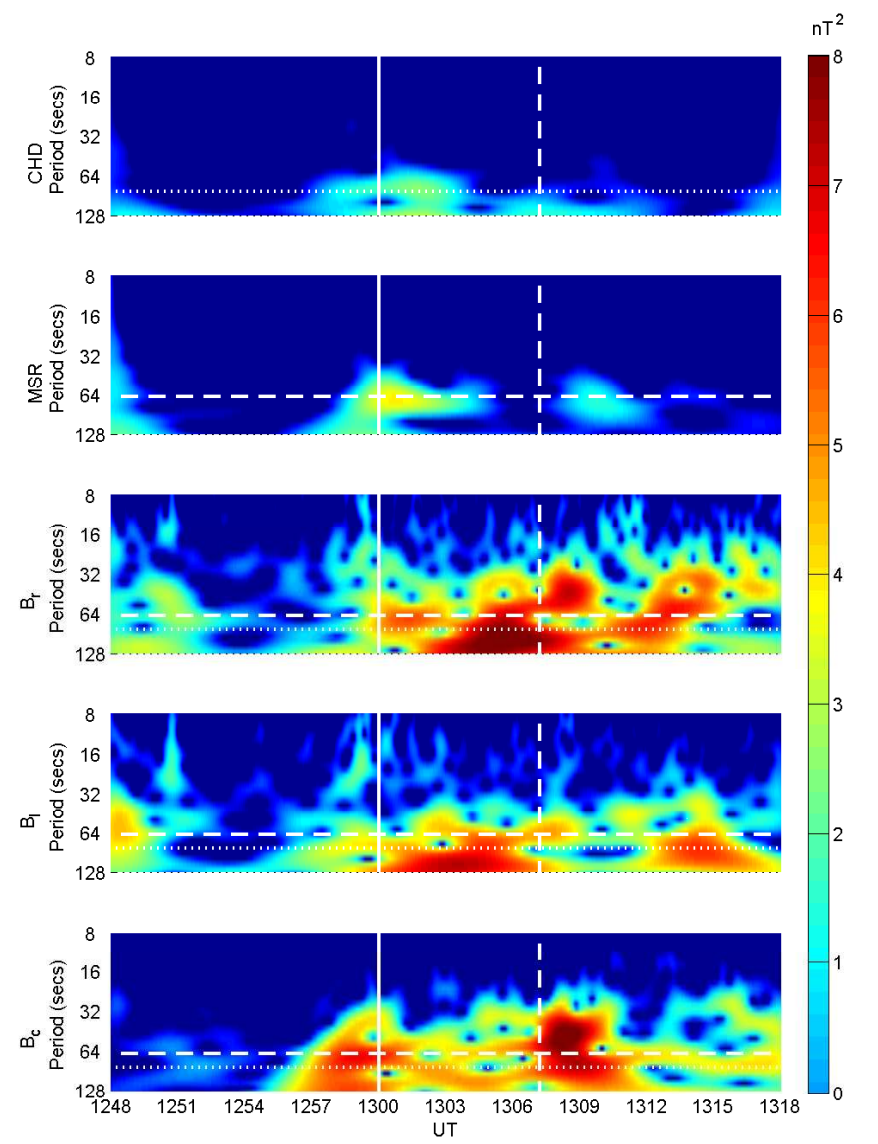

Fig. 4. Wavelet analysis for 6 October 2001 of the H-component from CHD and MSR and for the right- and left-hand polarized and compressional components of $\mathrm{C} 1$.

Interestingly, there is little correspondence between the waves observed on the ground and at Cluster, around Pi2 onset time. There is a small signature in $B_{\mathrm{r}}$, and clearly that belongs to a short dip in $B_{\mathrm{X}}$ near onset. This could be related to the fact that the footpoints of Cluster map to higher latitudes than that of ground station CHD.

However, $\sim 12$ min later (the vertical dashed line in Fig. 6) there are $\mathrm{Pi} 2$ waves at lesser amplitude in the ground data, and there is evidence in the Cluster data that there are similar oscillations observed in space. The power shows up both in $B_{1}$ and in $B_{\mathrm{r}}$ in the wavelet spectra, albeit at slightly longer periods, with the greatest power in $B_{1}$. Timing analysis of the Cluster data shows that the $\mathrm{Pi} 2$ waves are travelling at $v_{\mathrm{Pi} 2} \approx(-249,-32,-492) \mathrm{km} / \mathrm{s}$, which means tailward and southward. Cross-correlating the ground and space data we find that Cluster is lagging by $\sim 36 \mathrm{~s}$.

The wave power measured by CHD at long periods ( $\sim 90$ s) clearly shows up in both the left-handed and compressional component of the Cluster data.

The magnetometers on the ground show that the Pi2 waves at MRS are right-hand polarized, whereas those at CHD are

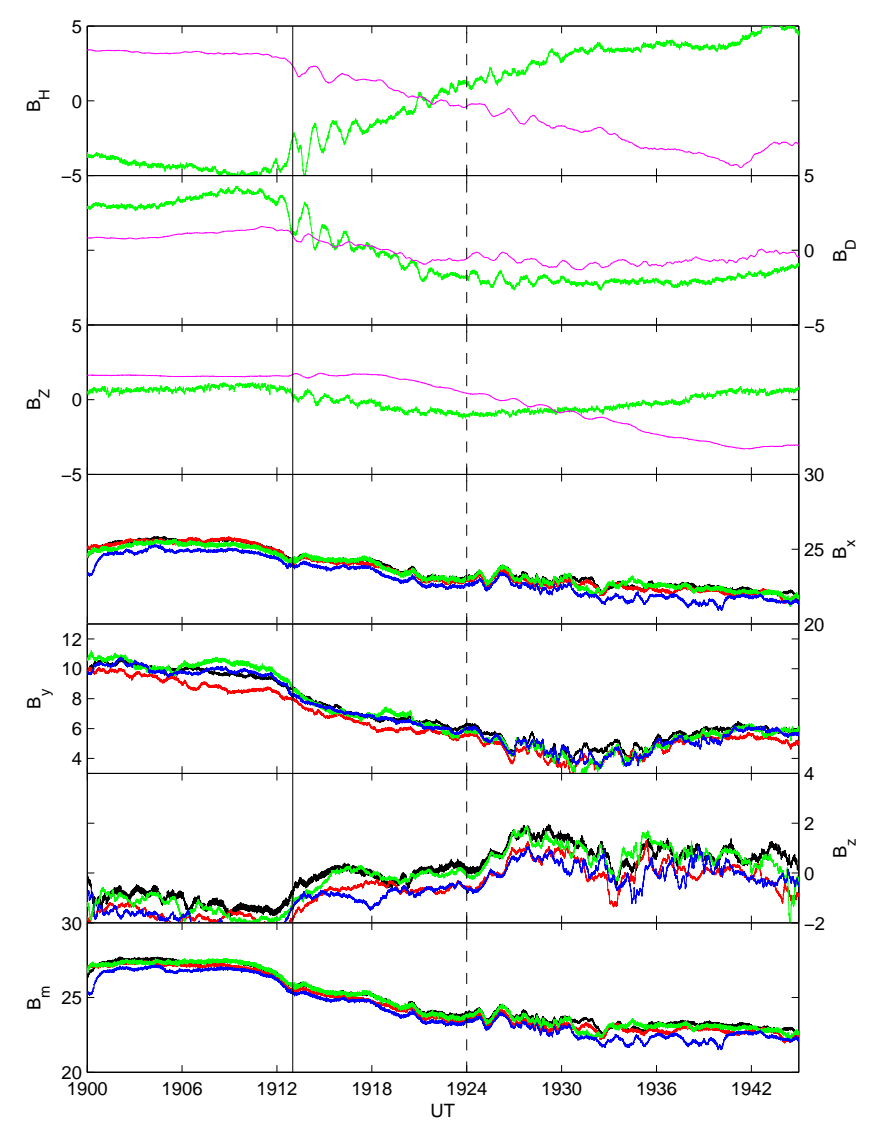

Fig. 5. Magnetic data for 26 July 2001. The top three panels show the H-, D- and Z-component of the magnetic field from the 210 meridian stations MSR (green) and CHD (magenta, divided by 10). The bottom three panels show the magnetic field measure by the Cluster spacecraft (in usual colours) in GSM coordinates. The dashed vertical line shows the time of Pi2 onset.

left-hand polarized, showing a change in polarization, probably caused by crossing the resonant field line between the two. The polarization of CHD agrees with that observed by the Cluster spacecraft.

\section{Discussion}

We have studied three conjunction events between Cluster and the 210 meridian chain near Pi2 onset. The Cluster spacecraft were always located in the lobe of the Earth's magnetotail during these events. Both on Earth as in space waves in the Pi2 frequency band were measured. The goal of this study was to investigate the propagation characteristics of the Pi2 waves in the tail, using the ability of Cluster to determine propagation direction through timing analysis. The three cases presented here show that in the lobes the Pi2 waves are all travelling tailward in the lobe. In Table 1 we give an overview of the location of the 210 meridian stations and the footpoints of the Cluster spacecraft. 

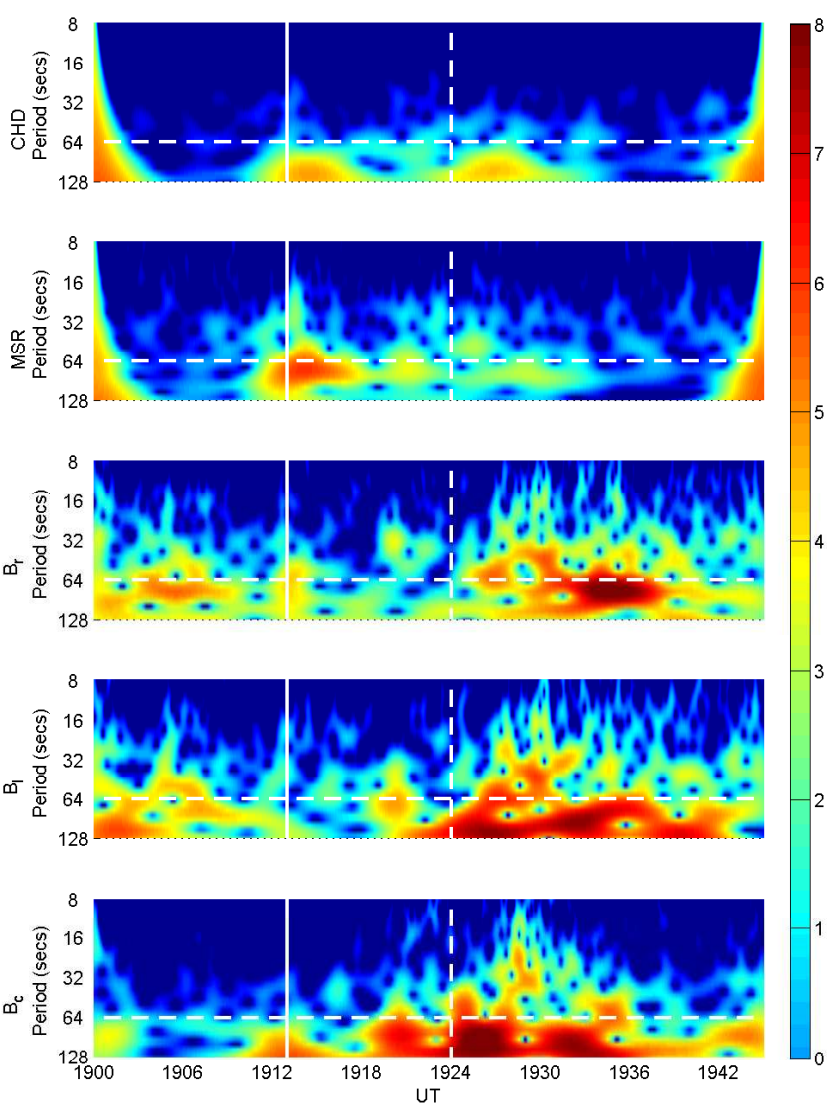

Fig. 6. Wavelet analysis for 26 July 2001 of the H-component from CHD and MSR and for the right- and left-hand polarized and compressional components of $\mathrm{C} 1$.

\subsection{Comparison of signals}

The wavelet analysis of the Cluster and 210 data has shown that waves in the Pi2 frequency band exist nearly simultaneously in both experiments. In order to compare whether Cluster and 210 measure the same waves, we have plotted the intervals of interest once more in Fig. 7. Here, we have shifted the 210 data according to the leading or lagging time with respect to Cluster, and the amplitude of the signal is adjusted to make the correspondences clear.

We show the transverse (blue) and compressional (red) component of the field measured by Cluster and the $B_{\mathrm{H}}$ measured by MSR (gray) and CHD (black). One can clearly see, that not only are the waves in both data sets present, as expected from the wavelet analysis, also specific details in the wave form correspond between ground and space observations. This shows, that not only is there similar wave activity, but both experiments are actually measuring the same waves. Therefore, the Pi2 waves, created on closed field lines in the dipolar region, find a way of transferring to higher latitude field lines. Uozumi et al. (2004) however, found from timing analysis between two latitudinally separated stations
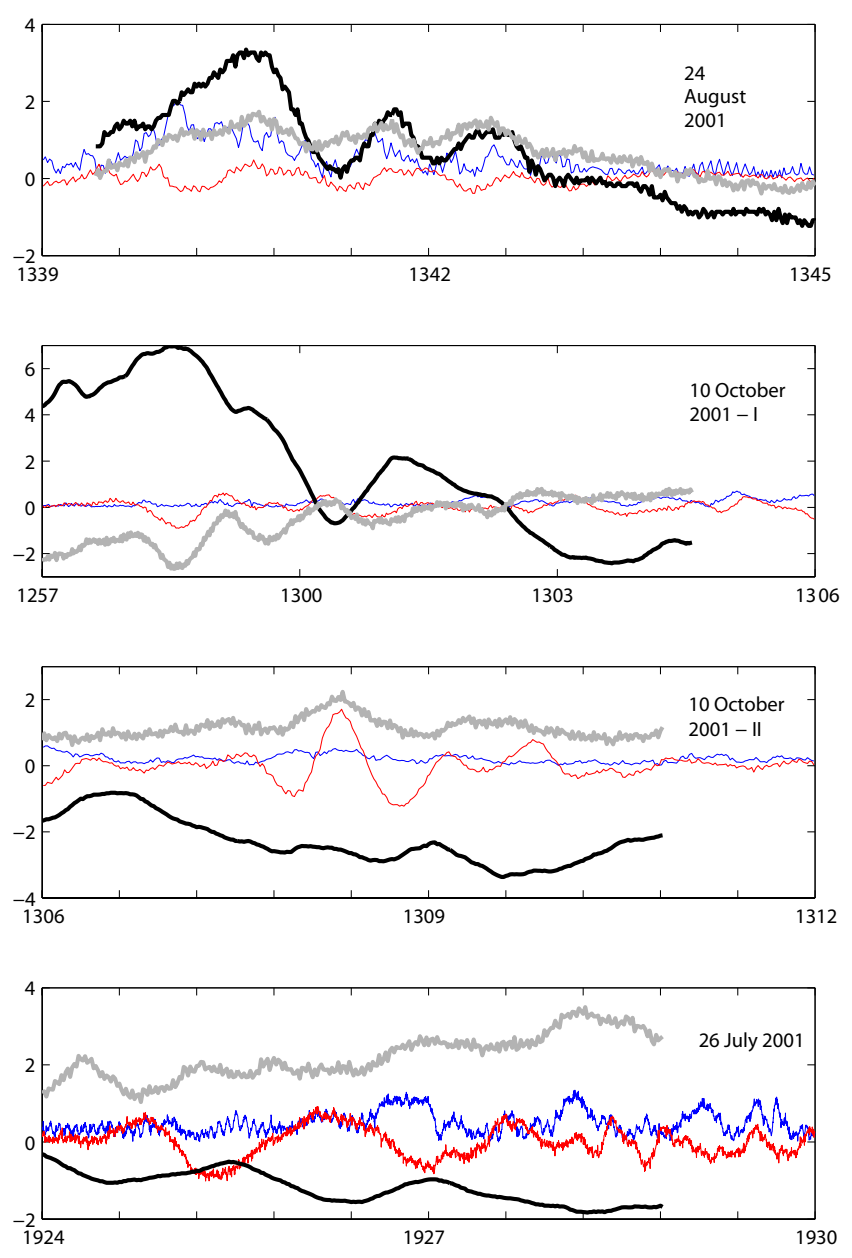

Fig. 7. Comparison of the wave signals for the intervals of interest, 24 August, 10 October ( 2 intervals) and 26 July. Shown are the transverse (blue) and compressional (red) magentic field of Cluster and the H-component of MSR (gray) and CHD (black). The gound station data have been shifted appropriately for the timing differences found.

Table 1. Tracing the footpoints of Cluster C1 to Earth in Geographical Magnetic coordinates using the T89 incorporated in the Orbital Visualization Tool (http://ovt.irfu.se), and the Geographical Magnetic coordinates of the 210 meridian stations.

\begin{tabular}{lrrrrr}
\hline Event/Station & 24 Aug 2001 & 6 Oct 2001 & 26 Jul 2001 & CHD & MSR \\
\hline N. Lat. & 73 & - & 79 & 71 & 44 \\
N. Lon. & 196 & - & 140 & 148 & 142 \\
L & & & & 5.55 & 1.6 \\
S. Lat. & -59 & -58 & -61 & & \\
S. Lon. & 152 & 121 & 136 & & \\
\hline
\end{tabular}

(KTM and TIK) that the station at highest latitude observed the Pi2 waves first. In our timing analysis no significant difference was found between the mid-latitude MSR and highlatitude CHD station. A possible explanation for this is if the 
generation of the Pi2 waves is localized in longitude and a combination of both latitudinal and longitudinal separation of these two stations. The Pi2 waves occur first at high latitude at MSR's longitude and then travel down in latitude and east in longitude to reach MSR and CHD at the same time respectively.

In detail we find that:

- For 24 August (Fig. 7 top panel) the waves observed by the ground stations follow rather well the blue (transverse) magnetic field measued by Cluster, although the amplitude is significantly different. This correspondence in polarization agrees with the conclusions obtained from the wavelet spectra.

- For 6 October (Fig. 7 middle 2 panels) the waves observed on the ground fit well with the compressional (red) component of the magnetic field measured by Cluster. Note that CHD is in anti-phase, whereas MSR is in phase with the Cluster data. This also fits well with the conclusions from the wavelet spectra.

- For 26 July (Fig. 7 bottom panel) there is good correspondence of CHD (black) with the compressional component (red) of Cluster, albeit in anti-phase. The signal from MSR (gray) seems to follow the transverse component (blue) of Cluster.

\subsection{Pi2 onset source}

With the first event we have shown that a compressional pulse travelling past Cluster towards the Earth might be responsible for the onset of the Pi2 waves. This is not an uncommon way of creating field line resonances. Indeed Keiling et al. (2001) discuss this very process, where a compressional wave source is located tailward from Polar, which couples resonantly to transverse field line resonance waves, a model proposed by e.g. Kivelson and Southwood (1986).

Checking the highest latitude station, CHD, there is evidence for a slightly earlier onset of the Pi2 activity, with the first peak just before 13:37 UT at $\sim 13: 36: 18$ UT. CHD is at an $L$-shell of 5.5, which the compressional pulse can easily reach with the measured velocity. A quick estimate for the resonant frequency at this $L$-shell can be made by:

$$
\begin{aligned}
T & =\oint \frac{d s}{v_{\mathrm{A}}} \equiv 2 l B^{-1} \sqrt{\mu_{0} m_{i} n_{0}}, \\
l & \approx \pi L-2,
\end{aligned}
$$

where we have assumed that the field line is a circle with diameter $L$, and have to subtract $2 R_{E}$ from its circumference as that is inside the Earth proper. With $v_{\mathrm{A}} \approx \alpha 10^{3} \mathrm{~km} / \mathrm{s}$ at $L=5.5$ one finds that $T \approx 194 / \alpha$ s., where $\alpha$ contains the local field strength and the plasma density $(|B| \approx 200 \mathrm{nT}$ and $n_{\mathrm{p}} \approx 0.1 \mathrm{~cm}^{-3}$ and $n_{\mathrm{O}^{+}} / n_{\mathrm{p}} \approx 0.5$ gives $\alpha \approx 3$ (generic values taken from Kivelson and Russell, 1995, chapter 10). This gives a period of $\sim 60 \mathrm{~s}$ for $L=5.5$, which is approximately the observed wave period at CHD and Cluster.

The resonant field line can couple with neighbouring field lines at larger $L$-shells, from which the waves may escape down the tail. In this way the escaping waves will have the same characteristics as those of the resonant field line.

Uozumi et al. (2000, 2004) studied the latitudinal propagation of Pi2s. Statistically, they find that the average time delay of the maximum of the Pi2 wave power between CHD and MSR is a few seconds, with CHD leading. However, the occurrence rate has a maximum around $20 \mathrm{~s}$. In their model (see also Itonaga and Yoshikawa, 1996), a compressional wave mode travels radially towards Earth, exciting shear Alfvén mode waves on the (dipolar) field lines, that propagate along the field towards the ground stations. In this way, because of the travel times of the different waves, the higher latitude stations observe the waves first.

If the source of the compressional wave is far down the tail, like in the first event of this paper, shear Alfvén waves can be generated on larger $L$, which could reflect at the ionosphere and travel back to the tail and be measured by Cluster. However, this mechanism need not lead to the same wave form observed in space and by the ground stations, which was discussed above.

\subsection{Propagation direction and polarization}

With Cluster in the magnetotail lobe (and possibly on open field lines in event 2) we find that the waves are all travelling away from Earth. This means that after the generation of the field line resonance, and the onset of Pi2, there must be an escaping mechanism, a leaking out of the waves, most likely at higher latitudes. The latter assumption arises from the polarization of the waves at CHD in the first event and the polarization of the waves at Cluster, which was the same, left-handed. This would agree with the polarization one would expect from Pi2 waves generated by the substorm current wedge as proposed by Lester et al. (1983), as Cluster is in the post-midnight hemisphere during the first event. Similarly for the third event on 26 July. For the second event 10 October we found that the polarization on the ground was highly elliptical and left-handed, which in the Cluster data showed up as compressional waves.

Regarding the polarization of the Pi2 waves at high latitude, Samson and Harrold (1983) show that it changes from predominantly couter-clockwise (as seen from above) equatorial of the westward surge region to clockwise (as seen from above) in the westward surge region at higher latitudes. This means that the polarization that we observe at the high-latitude station CHD is opposite from what Samson and Harrold (1983) found. The polarization characteristics of Pi2 waves have been also been discussed by Hopcraft and Smith (1989) theoretically for wave sources far down the tail. They show that the polarization of the waves is dependend on longitude, and that it reverses from left-hand polarized to 
right-hand when crossing the midnight meridian. This again, would agree with our ground observations.

\subsection{Comparison with previous observations}

How does this new information fit into what has been shown before in the tail? Recently various papers were published on Pi2 waves in the Earth's magnetotail.

Keiling et al. (2001) investigated conjugate observations by Polar and ground stations in oder to find a coupling between trapped and propagating waves to low-latitude field line resonances. In the Polar data (located between $3.7 \leq L \leq 4.1$ slightly duskward from the midnight meridian) they observe trapped and propagating fast modes and good coherence between Polar $B_{\mathrm{Z}}$ (field aligned component, compressional) and ground station $B_{\mathrm{H}}$. They present a model in which the source of the waves is located tailward of the spacecraft (see their Fig. 9), from which the fast mode waves originate, which then couple with closed field lines, creating field line resonances in the Pi2 band. It can be argued that the first event in this current paper is reminiscent of the model discussed by Keiling et al. (2001). Indeed, we also observe a compressional wave moving Earthward, and as it reaches the Earth there is Pi2 onset. So, as has been discussed before, a resonant coupling between compressional waves and transverse field line resonances can be a source for Pi2 waves.

Kim et al. (2005) studied Pi2 waves observed by Polar (located between $4.2 \leq L \leq 8.5$ at MLT between 0.3 and 0.7) and the low-latitude station Kakioka and mid-latitude stations Magadan and Zyryanka. In this case there is very good agreement between the $B_{\mathrm{Z}}$ (compressional) component of Polar and the $B_{\mathrm{H}}$ components of the ground stations. Polar is located in the magnetotail lobe in the first event and in the plasma sheet in the second event that is discussed. The one event in which Polar is in the lobe, Kim et al. (2005) find that $B_{\mathrm{H}}$ on the ground and $B_{\mathrm{Z}}$ in space show great similarity with out-of-phase signature, from which they conclude the waves must come from the same source.

Keiling et al. (2006) associate Pi2 waves with pulsed reconnection. This is the first paper to look at Pi2 waves with Cluster far down the tail at $X_{\mathrm{GSM}} \approx 16 R_{E}$ and in the lobe at $Z_{\mathrm{GSM}} \approx 2.5 R_{E}$. In this paper the $\mathrm{Pi} 2$ waves are interpreted as a series of of nightside flux transfer events passing by Cluster generated by pulsed reconnection further down the tail. With Cluster in the magnetotail lobe, it can be expected that they measured the same kind of Pi2 waves as the three events we present in this current paper. Unfortunately, although all four Cluster spacecraft observed the Pi2 waves (with small time delays) no effort was made to obtain the direction of propagation for these waves. However, they study the magnetic and electric field of the Pi2 waves and find that for 2 oscillations $B$ and $E$ are in phase, from which an Earthward propagation is deduced. From the ratio $E / B$ a propagation velocity is found between 600 and $800 \mathrm{~km} / \mathrm{s}$, which is then compared to the propagation velocity of a structure $1 \mathrm{~min}$ earlier, done by
Sergeev et al. (2005, see Fig. 10), which may or may not relate to the propagation velocity of the Pi2 waves. As has been shown in event 1 in this current paper, side-by-side magnetic structures need not propagate with the same velocity or direction. Therefore, it cannot be concluded whether our results are in (dis)agreement with those of Keiling et al. (2006).

Lately, Tokunaga et al. (2007) studied the global features of Pi2 and the relationship between the signatures observed at various latitudes. They decomposed the Pi2 waves at low latitudes into two components, which are labeled (A) and (B). The Pi2 (A) wave seems to be coherent over a wide range of latitudes, up to the high latitude stations, which map outside the plasma sphere. Therefore, Tokunaga et al. (2007) conclude that this component is a cavity mode but at larger scale than the usual plasmaspheric cavity resonance (see e.g. Yeoman and Orr, 1989), for which the plasmaspheric virtual resonance (Lee and Lysak, 1999) is responsible and Kim et al. (2005) showed that this mechanism is viable. The Pi2 (B) wave seems to be pertinent for high latitude stations and most likely related to the substorm current systems. Indeed, Tokunaga et al. (2007) show a possible immediate electric field coupling between the nightside and dayside auroral ionosphere. This can well be imagined to be the driving force behind the escaping Pi2 waves on high latitude field lines as observed in this current paper.

\section{Conclusions}

Pi2 waves in the Earth's magnetotail tail have now been well observed in various papers. We have found that Pi2 waves in the lobe of the magnetotail travel away from the Earth and have a polarization that is similar to that measured by highlatitude ground stations. The amplitude of the Pi2 waves in the magnetotail lobes is much smaller (up to more than 1 order of magnitude) than on the ground. However, one event (26 July) shows that, although clear Pi2 waves are observed on the ground, a similar signature is not found in the Cluster data in the magnetotail lobe, which may be linked to the spacecraft footpoints being significantly higher than the highest latitude ground station used in this paper.

Acknowledgements. Editor-in-Chief W. Kofman thanks one anonymous referee for her/his help in evaluating this paper.

\section{References}

Baumjohann, W., Treumann, R. A., Georgescu, E., Haerendel, G., Fornaçon, K.-H., and Auster, U.: Waveform and packet structure of lion roars, Ann. Geophys., 17, 1528-1534, 1999, http://www.ann-geophys.net/17/1528/1999/.

Collier, A. B., Hughes, A. R. W., Blomberg, L. G., and Sutcliffe, P. R.: Evidence of standing waves during a $\mathrm{Pi} 2$ pulsation event observed on Cluster, Ann. Geophys., 24, 2719-2733, 2006, http://www.ann-geophys.net/24/2719/2006/. 
Glaßmeier, K.-H., Baumjohann, W., Korth, A., and Gough, P.: High-latitude Pi2 pulsations, ELF intensity, and particle flux variations: A case study, Ann. Geophys., 6, 287-296, 1988, http://www.ann-geophys.net/6/287/1988/.

Harvey, C. C.: Spatial gradients and volumetric tensor, in: Analysis Methods for Multi-Spacecraft Data, edited by: Paschmann, G. and Daly, P., pp. 307-322, ESA, Noordwijk, 1998.

Hopcraft, K. I. and Smith, P. R.: Polarization characteristics of Pi2 oscillations, Planet. Space Sci., 34, 1259-1261, 1989.

Itonaga, M. and Yoshikawa, A.: The excitation of shear Alfvén wave and the associated modulation of compressional wave in the inner magnetosphere, J. Geomag. Geoelectr., 48, 1451-1459, 1996.

Keiling, A., Wygant, J. R., Cattell, C., Kim, K.-H., Russell, C. T., Milling, D. K., Temerin, M., Mozer, F. S., and Kletzing, C. A.: $\mathrm{Pi} 2$ pulsations observed with the Polar satellite and ground stations: Coupling of trapped and propagating fast mode waves to a midlattitude field line resonance, J. Geophys. Res., 106, 25 89125 904, 2001.

Keiling, A., Fujimoto, M., Hasegawa, H., Honary, F., Sergeev, V., Semenov, V. S., Frey, H. U., Amm, O., and Rème, H.: Association of $\mathrm{Pi} 2$ pulsations and pulsed reconnection: ground and Cluster observations in the tail lobe at $16 R_{E}$, Ann. Geophys., 24, 3433-3449, 2006, http://www.ann-geophys.net/24/3433/2006/.

Keiling, A., Angelopoulos, V., Larson, D., Lin, R., McFadden, J., Carlson, C., Bonnell, J. W., Moser, F. S., Glassmeier, K.H., Auster, H. U., Magnes, W., Mende, S., Frey, H., Roux, A., LeContel, O., Frey, S., Phan, T., Donovan, E., Russell, C. T., Mann, I., Liu, W., Li, X., Fillingim, M., Parks, G., Shiokawa, K., and Raeder, J.: Correlation of substorm injections, auroral modulations, and ground Pi2, Geophys. Res. Lett, 35, L17S22, doi:10.1029/2008GL033969, 2008.

Kepko, L. and Kivelson, M.: Generation of Pi2 pulsations by bursty bulk flows, J. Geophys. Res., 104, 25 021-25 034, 1999.

Kepko, L., Kivelson, M. G., and Yumoto, K.: Flow bursts, braking, and Pi2 pulsations, J. Geophys. Res., 106, 1903-1915, 2001.

Kim, K.-H., Lee, D.-H., Takahashi, K., Russell, C. T., Moon, Y.-J., and Yumoto, K.: Pi2 pulsations observed from the Polar satellite outside the plasmapause, Geophys. Res. Lett., 32, L18102, doi: 10.1029/2005GL023872, 2005.

Kivelson, M. G. and Russell, C. T.: Introduction to Space Physics, Cambridge University Press, New York, 1995.

Kivelson, M. G. and Southwood, D. J.: Coupling of global magnetospheric MHD eigenmodes to field line resonances, J. Geophys. Res., 91, 4345-4351, 1986.

Lee, D. H. and Lysak, R. L.: MHD waves in a three-dimensional dipolar magnetic field: A search for Pi2 pulsations, J. Geophys. Res., 104, 28 691-28 699, 1999.
Lester, M., Hughes, W. J., and Singer, H. J.: Polarization patterns of $\mathrm{Pi} 2$ magnetic pulsations and the substorm current wedge, J. Geophys. Res., 88, 7958-7966, 1983.

Mallat, S.: A Wavelet Tour of Signal Processing, Academic Press, San Diego, 1998.

Olson, J. V.: Pi2 pulsations and substorm onsets: A review, J. Geophys. Res., 194, 17 499-17 520, 1999.

Pashin, A. B., Glaßmeier, K.-H., Baumjohann, W., Raspopov, O. M., Yahnin, A. G., Opgenoorth, H. J., and Peillinen, R. J.: Pi2 magnetic pusations, auroral break-up, and the substorm current wedge, J. Geophys., 51, 223-233, 1982.

Samson, J. C. and Harrold, B. G.: Maps of the polarization of high latitude Pi2's, J. Geophys. Res., 88, 5736-5744, 1983.

Sergeev, V. A., Kubyshkina, M. V., Baumjohann, W., Nakamura, R., Amm, O., Pulkkinen, T., Angelopoulos, V., Mende, S. B., Klecker, B., Nagai, T., Sauvaud, J.-A., Slavin, J. A., and Thomsen, M. F.: Transition from substorm growth to substorm expansion phase as observed with a radial configuration of ISTP and Cluster spacecraft, Ann. Geophys., 23, 2183-2198, 2005, http://www.ann-geophys.net/23/2183/2005/.

Shiokawa, K., Baumjohann, W., Haerendel, G., Paschmann, G., Fennel, J. F., Friis-Christensen, E., Lühr, H., Reeves, G. D., Russell, C. T., Sutcliffe, P. R., and Takahashi, K.: High-speed ion flow, substorm current wedge, and multiple Pi2 pulsations, J. Geophys. Res., 103, 4491-4507, 1998.

Sigsbee, K., Cattell, C. A., Fairfield, D., Tsuruda, K., and Kokubun, S.: Geotail observations of low-frequency waves and high-speed earthward flows during substorm onset in the near magnetotail from 10 to $13 R_{E}$, J. Geophys. Res., 107(A7), 1141, doi:10.1029/ 2001JA000166, 2002.

Tokunaga, T., Kohta, H., Yoshikawa, A., Uozumi, T., and Yumoto, K.: Global feautures of Pi2 pulsation obtained by independent component analysis, Geophys. Res. Lett., 34, L14106, doi:10. 1029/2007GL030174, 2007.

Uozumi, T., Yumoto, K., Kawano, H., Yshikawa, A., Olson, J. V., Slovyev, S. E., and Veshinin, E. F.: Characteristics of energy transfer of $\mathrm{Pi} 2$ magnetic pulsations: Latitudunal dependence, Geophys. Res. Lett., 27, 1619-1622, 2000.

Uozumi, T., Yumoto, K., Kawano, H., Yoshikawa, A., Ohtani, S., Olson, J. V., Akasofu, S.-I., Solovyev, S. I., Vershinin, W. F., Liou, K., and Meng, C.-I.: Propagation characteristics of Pi2 magnetic pulsations observed at ground high latitudes, J. Geophys. Res., 109, A08203, doi:10.1029/2003JA009898, 2004.

Yeoman, T. K. and Orr, D.: Phase and spectral power of midlatitude $\mathrm{Pi} 2$ pulsations ovserved at ground high latitudes, Planet. Space Sci., 37, 1367-1383, doi:10.1016/0032-0633(89)90107-4, 1989. 